\title{
Several TOUGH2 Modules Developed for Site Characterization Studies of Yucca Mountain
}

\author{
Yu-Shu Wu and Karsten Pruess \\ Earth Sciences Division, Lawrence Berkeley National Laboratory, \\ Berkeley, CA 94720 , U. S. A.
}

\begin{abstract}
A comprehensive site characterization study has been conducted for the unsaturated zone (UZ) of Yucca Mountain to investigate its suitability as a potential high-level nuclear waste repository. Numerical modeling of moisture, gas, chemicals and heat flow, and their influence on the repository performance has been proven to be essential in understanding unsaturated-zone fluid movement, and the effects of hydrogeologic, geochemical and thermal conditions on various aspects of the overall waste disposal system. In these studies, the TOUGH 2 code has been used extensively as a main modeling tool because of its flexibility and robustness in handling multiphase, multi-component fluid and heat flow and chemical transport in porous/fractured rocks.
\end{abstract}

As part of the UZ modeling efforts at LBNL, several new modules of the TOUGH2 code have been developed to meet different needs of the site characterization studies. In this paper we will summarize some of these new TOUGH2 modules and their specific features in application to the Yucca Mountain project. These new TOUGH2 modules include (1) the effective continuum model (ECM) for treating fracture/matrix flow; (2) single-phase gas flow (EOS1G) in a water-air two phase flow system; and (3) a 3-D radionuclide transport module (T2R3D) for 3-D radionuclide or tracer transport in porous/fractured media under two-phase flow and nonisothermal conditions.

\section{Introduction}

Numerical modeling approaches used in the Yucca Mountain project for simulating multiphase fluid flow, heat transfer, and chemical transport processes involve coupled multiphase fluid and heat flow, and chemical component migration formulations based on finite difference, or finite element schemes. Available numerical simulators for variably saturated nonisothermal flow generally employ mathematical and numerical methods similar to those used in the oil industry or geothermal engineering. Since the oil and geothermal industries have different priorities than organizations dealing with nuclear waste repository assessment, the codes must account for a number of additional processes such as the slow and/or rapid movement of fluids in thick, highly heterogeneous, unsaturated systems over very long time periods. The improvement of the modeling capabilities for simulating field-scale problems has been subject to continuous research efforts, and considerable progress in this area has been made over the past two decades.

TOUGH2 (Pruess, 1991) has been used as a main modeling tool in the Yucca Mountain project due to its flexibility and robustness in handling multidimensional, multiphase, and multi-component fluid flow and heat transfer in both porous and fractured rocks. In addition, throughout the development of the TOUGH/TOUGH2 codes, a comprehensive verification and validation study has been conducted (Pruess, 1987 and 1991; Moridis and Pruess, 1992; Pruess et al., 1996). More recently, the TOUGH2 code has been updated and improved in the modeling studies associated with the site characterization of Yucca Mountain, and several new modules have been developed to add to the TOUGH2 family of codes (Wu et al., 1996). The correctness, validity, and capability of the TOUGH2 family of codes have been verified by comparing the simulation results against analytical solutions, and results from other numerical codes, laboratory experiments, and field tests.

In this paper, we will discuss three new TOUGH2 modules, developed during the site-characterization study at Yucca Mountain. These three new TOUGH2 modules are (1) effective continuum model (ECM) for treating fracture/matrix flow; (2) single-phase gas flow (EOS1G) in a water-air flow systems; and (3) a 3-D radionuclide transport module (T2R3D) for 3-D radionuclide or tracer transport in porous/fractured media under two-phase flow and non-isothermal conditions.

\section{Formulations}

ECM module was developed to simulate fluid flow, heat transfer and chemical transport in fractured porous media. This module can be used with any of the TOUGH2 EOS modules in simulation studies of flow and transport in fractured rock. The ECM formulation, as implemented in the TOUGH2 code (Wu et al., 1996), is based on the assumption that there is an approximate thermodynamic equilibrium 
(locally) between fracture and matrix at all times in the formation. For two-phase flow in a fractured porous medium, the condition of local thermodynamic equilibrium requires that temperatures, phase pressures, densities and viscosities in fracture and matrix systems are the same locally. Addition of the fluxes from Darcy's law for fracture and matrix gives the expressions for total flux through the fracture and matrix system:

$$
\mathbf{F}_{\beta}=-\mathrm{kk}_{\beta} \frac{\rho_{\beta}}{\mu_{\beta}}\left(\nabla \mathrm{P}_{\beta}-\rho_{\beta} \mathbf{g}\right)
$$

where $F_{\beta}$ is the total mass flux vector for phase $\beta ; \beta$ is phase index ( $\beta=1$ for liquid, and $\beta=\mathrm{g}$ for gas); $\rho_{\beta}$ is the density of phase $\beta ; \mu_{\beta}$ is the viscosity of phase $\beta$; $P_{\beta}$ is the pressure of phase $\beta$; and $g$ is the acceleration of gravity. In Equation (1), $k$ is the effective continuum permeability, defined as

$$
\mathrm{k}=\mathbf{k}_{\mathrm{m}}+\mathbf{k}_{\mathrm{f}}
$$

with $k_{m}$ being intrinsic continuum matrix permeability and $\mathbf{k}_{\boldsymbol{f}}$ intrinsic continuum fracture permeability. The effective continuum relative permeability, $k_{\beta}$, can be defined as

$$
k_{\beta}=\frac{k_{m} k_{\beta, m}+k_{f} k_{\beta, f}}{k_{m}+k_{f}}
$$

where $k_{\beta, m}$ and $k_{\beta, r}$ are relative permeability to phase $\beta$ in matrix and fracture, respectively. In these equations we have defined relative permeabilities relative to the total single phase permeability, $k$, so that $0 \leq k_{\beta} \leq 1$. The expression for the total flux of Equation (1) can be interpreted as refering to a single effective continuum with total effective phase permeability of $k_{m} k_{\beta, m}+k_{f} k_{\beta, f}$.

The numerical implementation of the ECM scheme for evaluating the effective continuum permeabilities is straightforward once we know fluid saturations in matrix and fracture, respectively. This can be achieved by introducing a fracture/matrix combined (or composite) capillary pressure $\left(P_{c}\right)$ curve (using table lookup, based on the individual fracture and matrix $P_{c}$ curves from input). Under local equilibrium conditions, the combined $P_{c}$ curve is

$$
P_{c}\left(S_{\beta}\right)=P_{c, m}\left(S_{\beta, m}\right)=P_{c, f}\left(S_{\beta, f}\right)
$$

as a function of a combined or average liquid saturation:

$$
S_{\beta}=\frac{S_{\beta, m} \phi+S_{\beta, f} \phi_{f}}{\phi_{m}+\phi_{f}}
$$

where $S_{\beta, f}$ and $S_{\beta, m}$ are saturations of phase $\beta$, and $\phi_{f}$ and $\phi_{\mathrm{m}}$ are porosities for fracture and matrix continuum, respectively.

During the Newton iteration in the TOUGH2 run, the liquid saturation obtained from the solution is exactly the combined saturation (5). The saturation value can be used in the combined $P_{c}$ curve to calculate the value of the combined capillary function. The fracture and matrix saturations can then be determined by inverting the $P_{c}$ functions of fracture and matrix, respectively. The ECM relative permeabilities are evaluated using Equation (3), where the relative permeabilities $k_{\beta, m}$ and $k_{\beta, r}$ have to be evaluated at saturations $S_{\beta, m}$ and $S_{\beta, r}$, respectively.

EOS1G (Air and Passive Water), the gas-flow-only module, was developed to efficiently simulate gas flow under partially saturated conditions. This module is especially useful in performing large-scale, 3-D pneumatic analyses and modeling studies of Yucca Mountain. The single-phase gas flow module uses the same description of the physical processes of multiphase flow in saturated and unsaturated heterogeneous media as in the standard TOUGH2 EOS3 module. It provides an accurate description of gas flow dynamics, and constitutive physical laws and properties affecting gas flow. It is capable of handling different flow geometries in one, two, and three dimensions, and rapidly changing surface atmospheric conditions.

The difference between the gas flow module and the standard version of the EOS3 module is that the gas flow module solves only one conservation equation for the air component, i.e. the water mass- and energy-balance equations used in the standard TOUGH 2 formulation are omitted. Liquid water and heat are assumed to be at steady state, but could be non-uniformly distributed within the system considered. EOS1G differs from the EOS3 module of TOUGH2 in (a) that the only primary thermodynamic variable is the gas pressure, (b) the water phase is treated as a passive phase with stable distribution, and (c) temperature does not change. The system is assumed to be at two-phase or singlephase gas conditions.

T2R3D (Radionuclide or Tracer Transport in 3-D) incorporates a full hydrodynamic dispersion tensor, based on a 3-D velocity field with a 3-D irregular grid, in a heterogeneous geological system. This new module of the TOUGH 2 code is designed to simulate processes of tracer/radionuclide transport using an irregular, 3-D integral finite-difference grid in nonisothermal, three-dimensional, multiphase, porous/fractured subsurface systems. The flow of 
water and air phases is treated using the TOUGH2 EOS3 formulation.

The major assumptions of the tracer transport module are: (a) a tracer or a radionuclide is present and transported only within the liquid phase, (b) transport mechanisms include molecular diffusion and hydrodynamic dispersion in addition to advection, and (c) first-order decay and linear adsorption onto rock grains are taken into account. The tracer or radionuclide is introduced as an additional mass component into the standard mass-conservation equations of TOUGH2, and the additional component equation is solved fully coupled with the conservation equations for water, air and heat. As a result, T2R3D can be used to study a wide range of transport problems, such as liquid or gas tracer transport, multi-component transport, strongly density-dependent processes, thermal effects, and nonlinear adsorption effects. Some of these applications, e.g. gas tracer and nonlinear absorping effects, may need minor additional modification to the code.

\section{Verification Examples}

\section{E CM}

In this problem we compare the simulation results using the ECM and those from the more rigorous dual-permeability modeling approach. The problem concerns one-dimensional vertical flow in an unsaturated zone of Yucca Mountain. The single vertical column grid is extracted directly from the 3-D site-scale model (Bodvarsson and Bandurraga, eds., 1996). The 1-D grid consists of 24 active grid blocks, 3 for the Tiva Canyon unit (TCw), 5 for the Paintbrush (PTn), 10 for the Topopah Springs (TSw), and 6 for the Calico Hills (CHn). These are the four major hydrogeological units at Yucca Mountain. An overlying and underlying boundary block represent the atmosphere and the water table conditions, respectively. The fracture system in the unsaturated zone of the mountain is also subdivided into the same four units, TCw, PTn, TSw, and $\mathrm{CHn}$. The properties of fractures are as listed in Table 1.

\section{Table 1. The fracture porosity and spacing data}

\begin{tabular}{ccc}
\hline Unit & Porosity & Spacing (m) \\
\hline TCw & $1.38 \mathrm{e}-3$ & 0.618 \\
PTn & $4.12 \mathrm{e}-3$ & 2.220 \\
TSw & $2.75 \mathrm{e}-3$ & 0.740 \\
CHn & $9.98 \mathrm{e}-4$ & 1.618
\end{tabular}

Since few fractures exist in the PTn unit, the effects of fractures on moisture flow in the PTn are ignored in this simulation, and the PTn formation is treated as a single porous medium rock. The same set of matrix and fracture properties (Wu et al., 1996) are used for both ECM and dual-permeability simulations.

The same boundary conditions are also specified for both models as Dirichlet-type conditions, i.e., constant pressures, temperatures and saturation. The surface boundary is subject to a constant water infiltration of $3.6 \mathrm{~mm} /$ year. The water infiltration on the ground surface is only added into the fracture elements on the top boundary for the dualpermeability model, while it is distributed between fracture and matrix in the ECM model because, realistically, infiltration is expected almost entirely through the fractures. However, the continuum approach of the ECM uses equilibrium partitioning for the infiltration. Also isothermal conditions were assumed.

Both models are nun to steady-state, and the simulation results are shown in Figure 1 for the steady-state liquid saturation profiles of matrix, obtained using the ECM and the dual-permeability models.

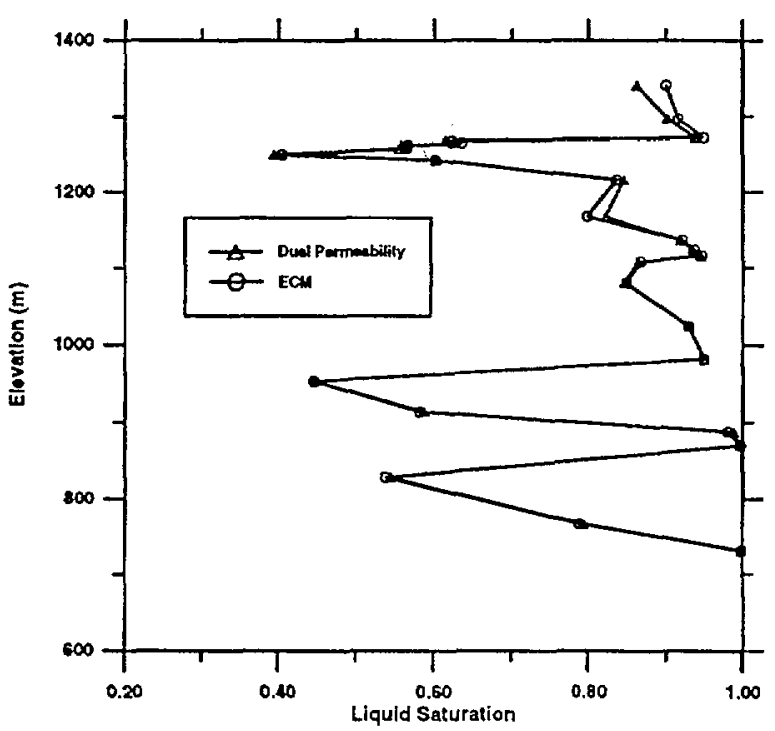

Figure 1. Steady-state liquid saturation profiles for fractures and matrix, obtained using the ECM and the dual-permeability models.

Figure 1 shows that almost identical results of matrix saturations are obtained from the two modeling approaches. The only differences between the two solutions may be noticed in the TCW unit (top), or along the interfaces between the PTn and the TSw (at about $1200 \mathrm{~m}$ elevation). These minor differences are believed to be due to the conceptual model, rather than the modeling approaches of two considerations. 
First, the water infiltration is imposed into fractures only on the top boundary in the dual-permeability simulation, while the ECM model puts the infiltration into both fracture and matrix systems. Second, the PTn unit is treated as a single porous medium, and this creates certain discontinuities in matrix/fracture vertical connections across the interfaces.

\section{EOS1G}

In this problem we compare the results of a transient gas flow simulation using the single-phase gas module (EOS1G) with those using the EOS3 module. A 1-D grid of 99 gridblocks is arranged vertically with one meter spacing between grid blocks. Steadystate initial conditions are gas static pressures with the bottom pressure of $90 \mathrm{kPa}$, a uniform temperature gradient from $30^{\circ} \mathrm{C}$ at the lower boundary to $10^{\circ} \mathrm{C}$ at the upper boundary, and variable liquid saturation from 0.99 at the lower boundary to 0.57 at the upper boundary. Variable liquid saturation distribution is initialized by running the TOUGH2 EOS3 and assuming $1 \mathrm{~mm} /$ year water infiltration into the upper boundary, capillary suction characteristic of Yucca Mountain tuffs (van Genuchten model with $\alpha=$ $0.0000167 \mathrm{~Pa}^{-1}$ and $\mathrm{m}=0.167$ ), and a Brooks-Corey relative permeability function. These parameters are unchanged for the comparison simulations, except that no liquid source term was used with the singlephase gas module. Other relevant parameters are 100 microdarcys absolute permeability and 0.01 porosity. The upper pressure boundary condition is temporally varying and is provided by tabular input to TOUGH2. All other boundaries are no-flow conditions. A constant time step of two hours is used in the simulations.

A comparison of pressure response at $30 \mathrm{~m}$ and $90 \mathrm{~m}$ below the upper boundary, simulated using the single-phase gas module (EOS1G) and EOS3, is shown in Figure 2. The agreement between the two modules is very good at $30 \mathrm{~m}$ and also at $90 \mathrm{~m}$ near the bottom boundary of the model. Actually, the results show that the agreement between the two modules is excellent for each gridblock at each time step.

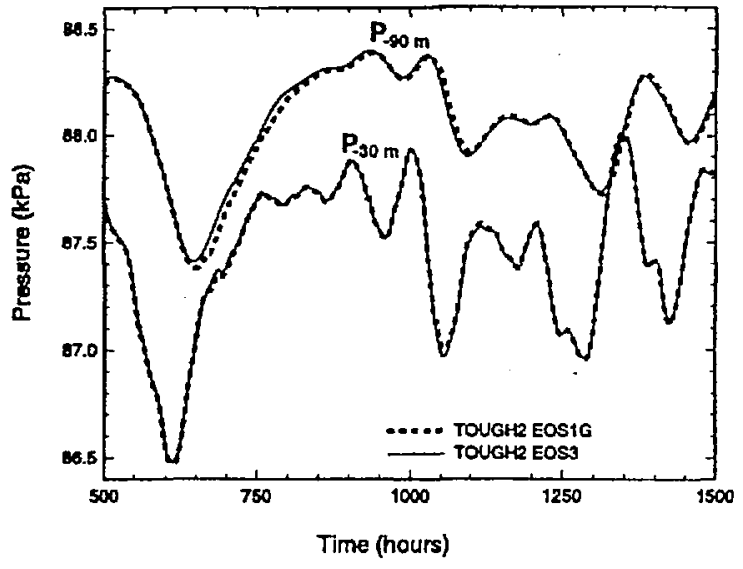

Figure 2. Time dependent pressures for the TOUGH2 simulations using the EOS3 module and the single-phase gas module (EOSIG).

\section{T 2R 3D}

The simulation presented here is designed to examine the accuracy of the T2R3D code in handling radionuclide transport in a multi-dimensional domain with hydraulic dispersion and molecular diffusion effects. The problem is similar to the one used by Oldenburg and Pruess (1993). It concerns twodimensional transport of a radionuclide in a homogenous, isotropic, saturated porous medium. An analytical solution for this problem is provided by Javandel et al. (1984) along with a code for calculating the 2-D concentrations. The analytical solution is used here to verify the T2R3D numerical solution. The model domain is rectangular, as shown in Figure 3. There is a steady-state, one-dimensional flow field along the $\mathrm{x}$-direction with pore velocity of $0.1 \mathrm{~m} /$ day. A radionuclide is introduced along a line source of length of 0.5 meter at $x=0$ with a constant concentration. Transport starts at $t=0$ from the line source by advection, hydraulic dispersion and diffusion. 


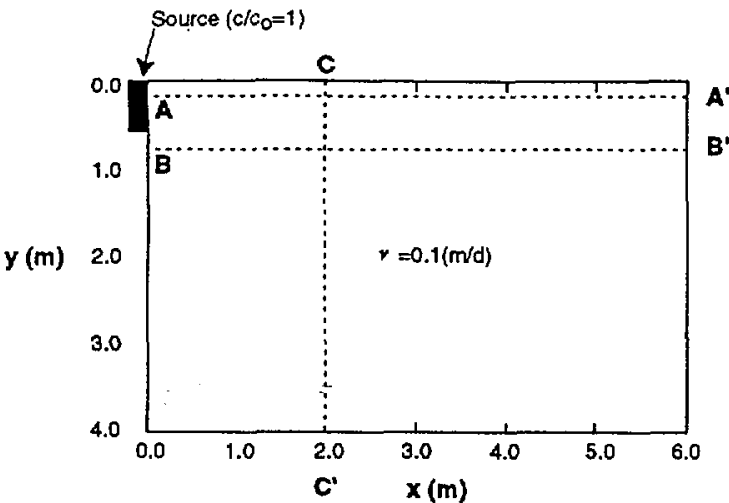

Figure 3. Schematic of the 2-D model domain for the two-dimensional radionuclide transport problem showing the velocity field and three cross sections for comparisons of the simulation results.

The T2R3D solution of this problem is accomplished by specifying on both the upstream boundary $(x=0)$ and downstream boundary $(x=6 \mathrm{~m})$ a constant pressure, which gives rise to a steady-state flow field of $0.1 \mathrm{~m} /$ day flow velocity: Also, in the T2R3D simulation, a uniform grid spacing was used for both $\mathrm{x}$ and $\mathrm{y}$ directions with $\Delta \mathrm{x}=\Delta \mathrm{y}=0.1 \mathrm{~m}$. The grid was generated using the TOUGH2 MESHMAKER. The system is kept at single liquid-phase and isothermal conditions. Air mass fraction is set to zero and a constant temperature of $25{ }^{\circ} \mathrm{C}$ is specified. Decay or adsorption effects are not included in the simulation. The properties used in the comparison study are: porosity $\phi=1$, tortuosity $\tau=1$, molecular diffusion coefficient $\mathrm{D}_{\mathrm{m}}=1.0 \times 10^{-10} \mathrm{~m}^{2} / \mathrm{s}$, longitudinal dispersivity $\alpha_{\mathrm{L}}$ is $0.1 \mathrm{~m}$, and transverse dispersivity $\alpha_{\mathrm{T}}$ is $0.025 \mathrm{~m}$. The liquid properties are internally generated by the code. The initial and boundary conditions for the radionuclide are: initially there is no radionuclide in the system; $\mathrm{X}^{\text {radionuclide }}$ $=1.0 \times 10^{-5}$ along the $0.5 \mathrm{~m}$ line source; and $\mathrm{X}^{\text {radionuclide }}$ $=0$ at the downstream boundary $(x=6 \mathrm{~m})$ at all times.

Comparison between the normalized radionuclide concentrations along the three cross-sections of the rock column from the T2R3D and the analytical solution is shown in Figures 4, 5, and 6 for time $=$ 20 days, respectively. The figures indicate that the T2R3D simulated concentration profiles in the twodimensional domain are in good agreement with the analytical solution. Figure 4 shows the radionuclide profile at $\mathrm{y}=0.15 \mathrm{~m}$ (cross section $\mathrm{A}-\mathrm{A}^{\prime}$ in Figure 3 ), indicating an excellent agreement between the two solutions along this cross section. Figure 5 shows the concentration profile at $y=0.75 \mathrm{~m}$ (cross section B-B'), just below the line source which extends from $y=0$ to $y=0.5 \mathrm{~m}$, and this figure displays both longitudinal and transverse dispersion effects.

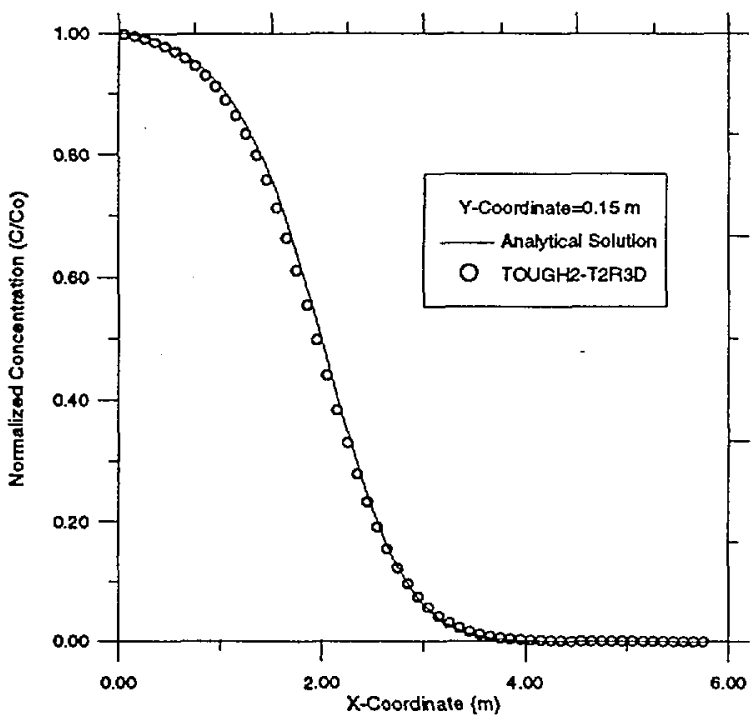

Figure 4. Comparison of radionuclide concentration profiles along cross section ( $\left.A-A^{\prime}\right)$ for analytical and numerical solutions at $t=20$ days.

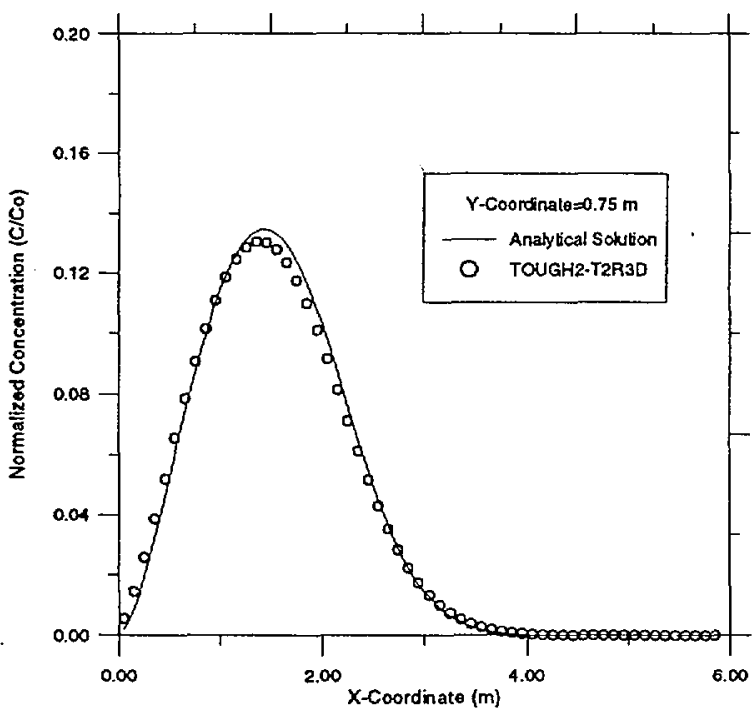

Figure 5. Comparison of radionuclide concentration profiles along cross section $\left(B-B^{\prime}\right)$ for analytical and numerical solutions at $t=20$ days.

As shown in Figure 5, the comparison is good even though small numerical errors are introduced in the numerical solution. Figure 6 gives the comparison of concentration profiles along the transverse direction (C-C') at $\mathrm{x}=2 \mathrm{~m}$, indicating an excellent agreement between the analytical and numerical solutions at this location. 


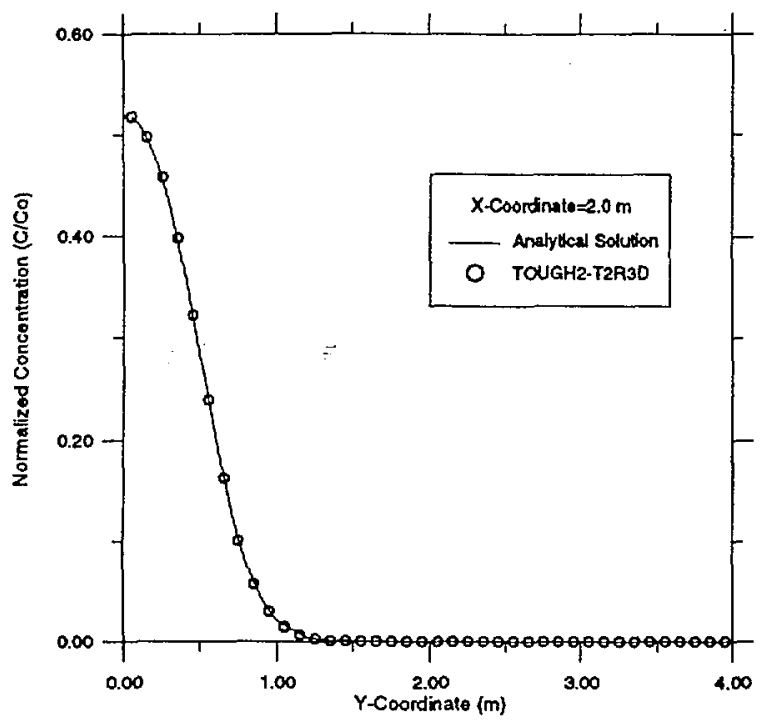

Figure 6. Comparison of radionuclide concentration profiles along cross section $\left(C-C^{\prime}\right)$ for analytical and numerical solutions at $t=20$ days.

\section{Summary}

This paper summarizes three new TOUGH2 modules, ECM, EOS1G and T2R3D developed during the site-characterization studies of Yucea Mountain. The model formulations are discussed briefly, and several verification examples are given. All the three modules are currently being used in the Yucca Mountain project.

\section{Acknowledgments}

The authors are grateful to H.H. Liu, A. Mishra, and $D$. Mangold for review of this paper and to M. Villavert for formatting this paper. This work was in part supported by the Director, Office of Civilian Radioactive Waste Management, U.S. Department of Energy, through Memorandum Purchase Order EA9013MC5X between TRW Environmental Safety Systems Inc. and the Ernest Orlando Lawrence Berkeley National Laboratory. The support is provided to Lawrence Berkeley National Laboratory through the U.S. Department of Energy Contract No. DE-AC03-76SF00098.

\section{References}

Bodvarsson, G.S., and Bandurraga, T.M. Eds., 1996. Development and Calibration of the ThreeDimensional Site-Scale Unsaturated Zone Model of Yucca Mountain, Nevada. Lawrence Berkeley
Laboratory Report, Berkeley, CA: Lawrence Berkeley National Laboratory.

Javandel, I.; Doughty, C.; and Tsang, C.F. 1984. Groundwater Transport: Handbook of Mathematic Models, American Geophysical Union, Water Resources Monograph 10, Washington, D.C.

Moridis, G.J. and Pruess, K. 1992. TOUGH Simulations of Updegraff's Set of Fluid and Heat Flow Problems, Report LBL-32611, Berkeley, CA: Lawrence Berkeley National Laboratory,

Oldenburg, C.M., and Pruess, K. 1993. A TwoDimensional Dispersion Module for the TOUGH2 Simulator, Report LBL-32505, Berkeley, California: Lawrence Berkeley National Laboratory.

Pruess, K., 1987. TOUGH User's Guide, Report NUREG/CR-4645 (Nuclear Regulatory Commission, Washington, D.C.), and Report LBL-20700, Berkeley, California: Lawrence Berkeley National Laboratory.

Pruess, K., 1991. TOUGH2 - A General-Purpose Numerical Simulator for Multiphase Fluid and Heat Flow, Report LBL-29400, Berkeley, California: Lawrence Berkeley National Laboratory.

Pruess, K., Simmons, A.; Wu, Y.S.; and Moridis, G.; 1996. TOUGH2 Software Qualification, Report LBL-38383, Berkeley, CA: Lawrence Berkeley National Laboratory.

Wu, Y.S., Ahlers, C.F.; Fraser, P.; Simmons, A.; and Pruess, K.; 1996. Software qualification of selected TOUGH2 modules, Report LBNL39490, Berkeley, CA: Lawrence Berkeley National Laboratory. 